\title{
The impact of contact lens wear on ocular surface mucins using a novel clinical fluorescence imaging system
}

DOI:

10.1016/j.clae.2019.08.004

\section{Document Version}

Accepted author manuscript

Link to publication record in Manchester Research Explorer

\section{Citation for published version (APA):}

Read, M., Navascues Cornago, M., Keir, N., Maldonado-Codina, C., \& Morgan, P. (2019). The impact of contact lens wear on ocular surface mucins using a novel clinical fluorescence imaging system. Contact Lens and Anterior Eye, 43(4), 378-388. https://doi.org/10.1016/j.clae.2019.08.004

\section{Published in:}

Contact Lens and Anterior Eye

\section{Citing this paper}

Please note that where the full-text provided on Manchester Research Explorer is the Author Accepted Manuscript or Proof version this may differ from the final Published version. If citing, it is advised that you check and use the publisher's definitive version.

\section{General rights}

Copyright and moral rights for the publications made accessible in the Research Explorer are retained by the authors and/or other copyright owners and it is a condition of accessing publications that users recognise and abide by the legal requirements associated with these rights.

\section{Takedown policy}

If you believe that this document breaches copyright please refer to the University of Manchester's Takedown Procedures [http://man.ac.uk/04Y6Bo] or contact uml.scholarlycommunications@manchester.ac.uk providing relevant details, so we can investigate your claim.

\section{OPEN ACCESS}




\title{
The impact of contact lens wear on ocular surface mucins using a novel clinical fluorescence imaging system
}

\author{
Michael L. Read ${ }^{\mathrm{a}, *}$, Maria Navascues Cornago ${ }^{\mathrm{a}}$, Nancy Keir ${ }^{\mathrm{b}}$, Carole Maldonado-Codina ${ }^{\mathrm{a}}$, Philip B. \\ Morgan $^{\mathrm{a}}$ \\ ${ }^{a}$ Eurolens Research, Division of Pharmacy and Optometry, Faculty of Biology, Medicine and Health, The University of \\ Manchester, Manchester, M13 9PL \\ ${ }^{b}$ CooperVision Inc., 6150 Stoneridge Mall Rd, Pleasanton, Calafornia, USA 94588
}

\begin{abstract}
Purpose: Fluorescein-labelled wheat germ agglutinin (F-WGA) acts as a marker for ocular surface mucins. This clinical study sought to investigate whether the degree of F-WGA fluorescence observed at the ocular surface differed between symptomatic contact lens wearers, asymptomatic contact lens wearers and non-contact lens wearers, using a novel imaging system. Methods: Twenty-five participants (10 symptomatic contact lens wearers, 10 asymptomatic contact lens wearers and 5 non-contact lens wearers) attended a single study visit. Photographs of the cornea, bulbar and tarsal conjunctiva were captured following application of F-WGA solution. Results: The imaging system captured high-resolution images of F-WGA fluorescence at the ocular surface. The degree of fluorescence differed between the ocular surface regions $(\mathrm{p}<0.001)$. A significant difference in fluorescence was observed between participant groups for the cornea $(\mathrm{p}=0.01)$, with both the symptomatic and asymptomatic contact lens wearers showing lower fluorescence than the non-lens wearers. F-WGA associated fluorescence appeared diminished in the lid wiper region of the symptomatic lens wearers, compared to the asymptomatic group $(\mathrm{p}=0.025)$. Conclusion: The use of F-WGA as a clinical marker for ocular surface mucins allows an improved understanding of their distribution across the ocular surface. Contact lens wear appears to negatively impact mucin density across the ocular surface, with the most marked effect on the cornea. F-WGA fluorescence appeared diminished in the lid wiper region for the symptomatic contact lens wearing group, indicating that mechanical interaction in this region may play a role in the aetiology of contact lens discomfort. Given the ability of F-WGA to disclose mucin distribution across the ocular surface it is likely to be a key clinical tool in furthering our understanding of (i) the aetiology of contact lens related discomfort, (ii) contact lens designs/materials to minimise interaction with the ocular surface and (iii) dry eye disease and other ocular surface diseases.
\end{abstract}

Keywords: Wheat germ aglutinin, Contact lens, Mucin, Lid wiper, Ocular surface, Comfort.

\section{Introduction}

At the interface between the tear film and the ocular surface, the stratified epithelium expresses a range of membrane-associated mucins, including MUC1, MUC4 and MUC16 [1]. These mucins form a dense glycocalyx layer, which acts as a barrier to prevent the penetration of pathogens, lubricates the motion of the eyelids, prevents adhesion of facing cells (i.e corneal epithelium and tarsal conjunctiva) during blinking/sleeping and provides a wettable surface to aid tear film adherence [2]. This structure is however

\footnotetext{
* Corresponding author.

Email addresses: michael.read-3@manchester.ac.uk (Michael L. Read), maria.navascuescornago@manchester.ac.uk (Maria Navascues Cornago), nkeir@coopervision.com (Nancy Keir), carole.m-codina@manchester.ac.uk (Carole Maldonado-Codina), philip.morgan@manchester.ac.uk (Philip B. Morgan)
} 
vulnerable to damage and is known to be altered by contact lens wear [3] and a range of ocular surface diseases including allergy, non-autoimmune dry eye, autoimmune dry eye and infection [4].

It is well documented that wheat germ agglutinin (WGA) is able to bind to N-acetyl-D-glucosamine and sialic acid [5, 6], which are found in the human cornea [6]. By attachment of a fluorescent conjugate, the presence of WGA can be visualised using fluorescence microscopy [7]. Recent work has shown that fluorescein-labeled WGA (F-WGA) can act as a clinical marker for the presence of the ocular surface mucins and the glycocalyx [8-10]. However, due to the low intensity of fluorescence observed with FWGA, this approach has previously required the use of a fluorophotometer (an ophthalmic instrument which measures fluorescent intensity across a $2 \mathrm{~mm}$ diameter region) and thus has lacked the spatial resolution of a conventional imaging system. In addition, previous F-WGA research has focused on the cornea and bulbar conjunctiva, whereas recent work has highlighted the importance of the tarsal conjunctiva and in particular the lid wiper region to ocular comfort [11-14]. The objectives of this work were (i) to develop an imaging system to characterise F-WGA staining at the ocular surface, and (ii) to use this instrument to investigate whether F-WGA fluorescence at the ocular surface differed between symptomatic contact lens wearers, asymptomatic contact lens wearers and non-contact lens wearers.

\section{Methods}

\subsection{Custom imaging system}

In the development of a custom imaging system to characterise F-WGA fluorescence at the ocular surface, a number of technical challenges had to be overcome, including the low level of fluorescence produced by F-WGA, the curvature of the ocular surface, continuous microscopic eye movements and photobleaching of the fluorophore (reduction in fluorescence due to excessive light exposure). The imaging system used a high sensitivity back-illuminated CMOS (complementary metal-oxide-semiconductor) imaging sensor (8.4 $\mathrm{\mu m}$ pixel pitch), combined with small aperture macro optics and flash illumination to obtain the images captured in this study. A digital camera (Sony $\alpha 7 \mathrm{~S}$ II, Sony Europe Ltd., Weybridge, UK) was mounted on an ophthalmic instrument base (Takagi Ltd., Manchester, UK) incorporating a chin rest, allowing the camera to be aligned with the participant's eye during clinical imaging. To allow high resolution imaging across the curved ocular surface, a macro lens was selected (Sony FE $90 \mathrm{~mm}$ Macro lens and $26 \mathrm{~mm}$ extension tube, Sony Europe Ltd., Weybridge, UK) and a small aperture employed (f/20) to optimise depth of field. To minimise image blur whilst ensuring sufficient fluorescence from the F-WGA, a macro flash illumination source (Canon MT-24EX, Canon Europe Ltd., Uxbridge, UK) and a short exposure time $(1 / 25$ seconds) were used. Excitation optical bandpass filters (MF475-35 $\varnothing 50 \mathrm{~mm}$, Thorlabs Ltd., Ely, UK) were positioned in front of both flash heads and an emission optical bandpass filter (MF530-43 $\varnothing 50 \mathrm{~mm}$, Thorlabs Ltd., Ely, UK) positioned over the camera objective with custom mounts. These optical bandpass filters were selected to match the spectral characteristics of F-WGA (excitation of $494 \mathrm{~nm}$ and emission of $518 \mathrm{~nm}$ ). The flash was used in a manual mode $(1 / 1)$, with the flash heads positioned on each side of the objective and rotated 45 degrees inwards to ensure even illumination. An additional low intensity LED lamp (LIU470A, Thorlabs Ltd., Ely, UK) was mounted below the objective with an excitation optical bandpass filter (MF475-35 $\varnothing 25 \mathrm{~mm}$, Thorlabs Ltd., Ely, UK) to allow camera alignment and focusing, prior to image capture. A live video feed from the camera was displayed on a wall-mounted monitor to aid in this process. To ensure that the illumination was consistent, images of a fluorescent calibration slide were captured (FSK5 Green, Thorlabs Ltd., Ely, UK). The imaging system was calibrated with a concentric square target (R3L3S3P, Thorlabs Ltd., Ely, UK). Prior to the clinical study, testing was undertaken to investigate whether use of the custom imaging system resulted in any significant F-WGA photo bleaching. In this work, F-WGA solution was dispensed between two glass microscopy slides with a $10 \mu \mathrm{m}$ separation to model its distribution within the tear film. A series of 7 images of this F-WGA film were then captured (10 seconds between each captured image) and the intensity of fluorescence was then assessed for each image using custom image analysis software.

\subsection{F-WGA preparation}

Fluorescein labelled-wheat germ agglutinin (F-WGA) (ThermoFisher Scientific Inc., UK) was suspended in sterile ophthalmic saline (Ami-dose, Albatron Ltd., UK) to form a $5 \%$ suspension (as previously described 
by Mochizuki et al.[10]). This suspension was then passed through a sterile $0.22 \mu \mathrm{m}$ Millex syringe filter (Merck Inc., Darmstadt, Germany) into a sterile 1.5ml microcentrifuge tube (Sigma-Aldrich Ltd., UK). The microcentrifuge tube was stored for up to one week in a laboratory refrigerator $\left(5 \pm 3^{\circ} \mathrm{C}\right)$. After one week the microcentrifuge tube and contents were discarded and a new suspension prepared. This F-WGA solution was used for both the laboratory imaging (described in Section 2.1) and the clinical study.

\subsection{Clinical study}

This was a prospective, controlled, non-randomised, open-label, parallel group clinical study which used the custom imaging system to quantify the fluorescence associated after the application of F-WGA. This technique was used to compare mucin distribution at the ocular surface between a group of symptomatic and asymptomatic contact lens wearers, in addition to a control group of non-contact lens wearing participants. In total 25 participants were recruited, with 10 symptomatic contact lens wearing participants (defined as a CLDEQ-8 questionnaire [15] score $\geqslant 14$ and a difference between contact lens wear time and comfortable wear time $\geqslant 2$ hours), 10 asymptomatic contact lens participants (defined as a CLDEQ-8 questionnaire score $\leqslant 8$ and a difference between contact lens wear time and comfortable wear time $\leqslant 1$ hour) and 5 non-contact lens wearing participants (defined as no more than one day of contact lens wear experience in the last 2 years and a DEQ-5 questionnaire [16] score $<8$ ). As this work represented the first time the custom imaging system had been deployed for the assessment of F-WGA no a priori power analysis was possible. These subject numbers were considered to be reasonable to (a) determine if the imaging system could be used, (b) to provide some initial evaluation of the utility of this system to compare symptomatic and asymptomatic contact lens wearers with non-wearers and (c) to provide a dataset for power analysis for future work in this area.

All participants provided written informed consent before inclusion in the study. The study was conducted in accordance with the principles of the Declaration of Helsinki and The University of Manchester Research Ethics Committee provided ethical approval. Individuals with a history of ocular/systemic disorders that would normally contraindicate contact lens wear, history of ocular surgery, use of topical ophthalmic medication, corneal distortion, pregnant or breastfeeding, a history of anaphylaxis or severe allergic reaction or any infectious or immunosupressive disease that would pose a risk to study personnel, were excluded.

Each participant attended for a single study visit. Here, the contact lens-wearing participants attended wearing their habitual contact lenses, having worn them for at least 4 hours. The non-contact lens wearing participants attended clinic a minimum of 4 hours after waking. Subjective ocular comfort was assessed using a 0-100 visual analogue scale $(0=$ very uncomfortable / $100=$ excellent comfort $)$. If the participant was a contact lens wearer, habitual lens fit was assessed (horizontal and vertical centration, corneal coverage and movement), details of the habitual lenses/care system were recorded and their contact lenses were then removed. Corrected high contrast distance $\log M A R$ visual acuity was then recorded. Slit lamp biomicroscopy was performed with clinical signs graded to the nearest 0.1 unit using the Efron Grading Scales (without sodium fluorescein assessment to avoid adversely influencing F-WGA imaging). Baseline photographs were then captured (in a randomised order) of seven ocular surface regions (i) the cornea (primary gaze with lids retracted using an Eyegenie external lid retractor (Bernell Inc., Mishawaka, IN)), (ii to v) the bulbar conjunctiva (in superior, inferior, nasal and temporal gaze) and (vi and vii) the tarsal conjunctiva (following evertion of the upper and lower eye lids), using the custom imaging setup described in Section 2.1. A 5 pl droplet of F-WGA solution was applied to the temporal conjunctiva of one eye, followed by the same process in the other eye three minutes later (approximately 15 minutes after contact lens removal or after slit lamp biomicroscopy in the case of a non-contact lens wearer). Approximately five minutes and 30 minutes after FWGA application, photographs were again captured of the cornea, bulbar conjunctiva and tarsal conjunctiva in a randomised order (a single image captured for each of the seven regions previously imaged). Subjective comfort was also assessed at these two time points using a 0-100 visual analogue scale. Following imaging at the 30 minute time point, slit lamp biomicroscopy was undertaken including assessment with sodium fluorescein. Corrected high contrast distance $\log$ MAR visual acuity was recorded and the participant was exited from the clinical study. 


\subsection{Image analysis}

During the clinical study, seven ocular surface regions were imaged for each of the participants eyes: cornea, bulbar conjunctiva (in superior, inferior, nasal and temporal gaze) and tarsal conjunctiva (upper and lower everted lids). Captured images were exported and coded by the clinical investigator. Image analysis was undertaken by a different masked investigator who analysed all clinical images in a randomised order. For each image, custom MATLAB image analysis software was used to isolate the region of interest and calculate the degree of fluorescence (assessed as the mean pixel value of the RGB image). For the image captured of the cornea, the region of interest was the entire visible corneal region. For the four images captured of the bulbar conjunctiva (with the subject in superior, inferior, nasal and temporal gaze), the region of interest was the entire visible bulbar conjunctival in each image (excluding the tear film meniscus and areas obscured by the lashes). For the images captured of the everted tarsal conjunctiva, the region of interest was the entire tarsal conjunctiva visible in each image. To calculate the degree of fluorescence attributable to the F-WGA, the baseline fluorescence was subtracted from the 5-minute and 30-minute post-FWGA application images. This value was termed the Background Subtracted Intensity (BSI). An image analysis algorithm was also developed to characterise fluorescence in the lid wiper region of the upper and lower eye lid. The masked image analysis investigator was instructed to draw a line along the line of Marx and the custom image analysis algorithm (MATLAB, The MathWorks, Natick, MA), then calculated the average BSI over the lid wiper region (extending $0.6 \mathrm{~mm}$ from the line of Marx as detailed by Efron et. al. [17]). The BSI of a reference region of visible tarsal conjunctiva (all of the visible tarsal conjunctiva $>1 \mathrm{~mm}$ from the line of Marx) was then subtracted from the BSI of the lid wiper region to allow an assessment of relative fluorescence in the lid wiper region. For this metric of relative lid wiper fluorescence, a value above 0 indicated elevated F-WGA fluorescence in the lid wiper region, whereas a value below 0 indicated a depressed fluorescence in the lid wiper region. A further image analysis algorithm was developed to characterise fluorescence in the corneal limbal region. The investigator was instructed to mark the corneal limbus on the primary gaze image. The algorithm then performed a series of radial pixel intensity scans centred on the cornea. The algorithm then averaged these scans to produce an averaged radial scan intensity profile for each primary gaze image. As with the regional analysis, the baseline fluorescence profile was subtracted from the F-WGA fluorescence profile, to generate the background subtracted profile for both the lid wiper region and the corneal limbus region.

\subsection{Statistical approach}

Habitual contact lens wearing time, comfortable hours of lens wear and CLDEQ-8 data were analysed using a Student's t-test. Contact lens fitting data were analysed using a chi-square test. Biomicroscopy data were assessed using a linear mixed model, with participant group, participant ID (random effect), eye (nested within participant ID) and assessment period (pre or post-F-WGA imaging) as factors of interest. Subjective comfort scores (0-100 VAS) were assessed using a linear mixed model, with participant group, participant ID (random effect), age and assessment period (pre or post-F-WGA imaging) as factors of interest. Background-subtracted ocular surface fluorescence intensity data were assessed using a linear mixed model, with participant group, participant ID (random effect), age, eye (nested within participant ID), imaging time point and ocular surface region as factors of interest. Visual acuity data were assessed using a linear mixed model with assessment period, participant group and participant ID (random effect) as factors of interest. The difference in fluorescence between the lid wiper region and the reference region of the tarsal conjunctiva were assessed using a linear mixed model, with participant group, participant ID (random effect), age, eye (nested within participant ID), imaging time point and eyelid as factors of interest. For all linear mixed models, interaction factors were initially included in the model, but were removed if the factors were not significant at $p>0.2$. Tukey post-hoc analysis was performed where appropriate. A p-value of less than 0.05 was considered statistically significant. All data were analysed using JMP 14, Version 14.3 (SAS Institute Inc. Cary, NC, USA). 


\section{Results}

\subsection{Clinical assessment}

Table 1 details the participant demographics, habitual lens information, typical lens wear time and typical subjective ocular comfort for the three participant groups. A statistically significant difference was observed between the two contact lens wearing groups, for the comfortable wear time $(\mathrm{F}=12.4, \mathrm{p}=0.002)$ and uncomfortable wear time (i.e. total hours per day - comfortable hours per day) $(\mathrm{F}=44.5, \mathrm{p}<0.0001)$. No significant difference was observed between the subject groups for subject age $(\mathrm{F}=3.2, \mathrm{p}=0.06)$, days per week of contact lens wear $(\mathrm{F}=3.2, \mathrm{p}=0.09)$ or hours per day of contact lens wear $(\mathrm{F}=3.0, \mathrm{p}=0.10)$. A statistically significant difference was seen between the two contact lens wearing groups for the CLDEQ8 score $(\mathrm{F}=193.7, \mathrm{p}<0.0001)$. All habitual contact lenses were seen to fit acceptably, with no difference observed between the symptomatic and asymptomatic contact lens wearing groups for the percentage of optimum lens fits $\left(\chi^{2}=1.9, \mathrm{p}=0.17\right)$.

\begin{tabular}{lccc}
\hline Parameter & Symptomatic & Asymptomatic & No lens wear \\
\hline Gender & 9 female / 1 male & 7 female / 3 male & 3 female / 2 male \\
Age & $39.2 \pm 13.6$ & $28.4 \pm 4.7$ & $40.6 \pm 13.8$ \\
Habitual lens material & 8 hydrogel / $2 \mathrm{SiH}$ & 5 hydrogel / $5 \mathrm{SiH}$ & - \\
Habitual lens modality & $8 \mathrm{DD} / 2 \mathrm{FR}$ & $5 \mathrm{DD} / 5 \mathrm{FR}$ & - \\
Days of CL wear per week & $4.3 \pm 2.2$ & $5.9 \pm 1.9$ & - \\
Hours of CL wear per day & $10.2 \pm 1.8$ & $13 \pm 4.8$ & - \\
Comfortable hours of lens wear per day & $6.3 \pm 2.4$ & $12.6 \pm 5.1$ & - \\
Uncomfortable Wear time (hours per & $3.9 \pm 1.6$ & $0.4 \pm 0.5$ & - \\
day - comfortable hours per day) & & & $2.0 \pm 1.6$ \\
CLDEQ-8 (DEQ-5 for non-lens wearers) & $20.7 \pm 2.5$ & $5.3 \pm 2.5$ & \\
\hline
\end{tabular}

Table 1: Participant demographics and habitual contact lens information for the three participant groups (mean \pm standard deviation).

High contrast visual acuity and subjective ocular comfort scores prior to and following the F-WGA imaging procedure are shown in Table 2. A statistically significant improvement in visual acuity was observed following the F-WGA imaging procedure $(\mathrm{F}=6.1, \mathrm{p}=0.02)$, although this did not reach the level of clinical significance. Subjective comfort scores (0-100 VAS) prior to F-WGA application showed a significant difference between the participant groups $(\mathrm{F}=9.8, \mathrm{p}=0.0009)$, with a Tukey post-hoc analysis demonstrating a significantly lower comfort for the symptomatic group, than both the asymptomatic and non-lens wearing group. The linear mixed model analysis for the entire 0-100 VAS subjective comfort data set highlighted that the effect of participant group $(\mathrm{F}=5.1, \mathrm{p}=0.02)$, assessment period $(\mathrm{F}=6.04, \mathrm{p}=0.02)$ and the interaction term group*assessment period $(\mathrm{F}=6.6, \mathrm{p}=0.006)$ reaching statistical significance; with the effect of age not reaching significance $(\mathrm{F}=3.4, \mathrm{p}=0.08)$. A Tukey post-hoc analysis demonstrated a statistically significant reduction in comfort following F-WGA application. A Tukey post-hoc analysis on the interaction term (group*assessment period), demonstrated that the change in comfort between the entrance and exit measures was not consistent between the participant groups, with the comfort of the lens wearing groups not changing significantly prior to and following F-WGA application, whereas the non-lens wearing group showed a significant reduction in subjective comfort.

\begin{tabular}{lccc}
\hline Parameter & Symptomatic group & Asymptomatic group & No lens wear group \\
\hline Entrance VA & $-0.07 \pm 0.09$ & $-0.03 \pm 0.16$ & $0.01 \pm 0.19$ \\
Exit VA & $-0.12 \pm 0.07$ & $-0.06 \pm 0.15$ & $0.00 \pm 0.17$ \\
Entrance 0-100 VAS comfort & $63.8 \pm 20.0$ & $87.5 \pm 10.9$ & $96.0 \pm 8.9$ \\
Post F-WGA 0-100 VAS comfort & $72.1 \pm 17.4$ & $78.2 \pm 18.0$ & $68.4 \pm 19.5$ \\
\hline
\end{tabular}

Table 2: Visual acuity and subjective comfort scores for the three participant groups (mean \pm standard deviation).

Slit lamp biomicroscopy grading scores were statistically similar when assessed prior to and following the F-WGA imaging procedure (Table 3). Due to the sensitivity of the imaging system to ocular surface 
fluorescence, sodium fluorescein was applied only on completion of F-WGA imaging procedure, with the degree of staining observed typical of that reported in the literature following contact lens wear and on the bare eye, for the non-contact lens wearing participants [18].

\begin{tabular}{cccc}
\hline Parameter & Pre-imaging grading & Post-imaging grading & Linear mixed model \\
\hline Conjunctival hyperaemia & $0.64 \pm 0.22$ & $0.66 \pm 0.26$ & $\mathrm{~F}=0.5, \mathrm{p}=0.51$ \\
Limbal hyperaemia & $0.54 \pm 0.29$ & $0.54 \pm 0.28$ & $\mathrm{~F}=0.1, \mathrm{p}=0.71$ \\
Corneal vascularisation & $0.02 \pm 0.07$ & $0.02 \pm 0.07$ & $\mathrm{~F}<0.0001, \mathrm{p}=1.0$ \\
Corneal oedema & $0.00 \pm 0.00$ & $0.00 \pm 0.00$ & $\mathrm{~F}<0.0001, \mathrm{p}=1.0$ \\
Blepharitis & $0.18 \pm 0.39$ & $0.17 \pm 0.37$ & $\mathrm{~F}=0.6, \mathrm{p}=0.56$ \\
Corneal staining & - & $0.57 \pm 0.49$ & - \\
Conjunctival staining & - & $0.48 \pm 0.65$ & - \\
\hline
\end{tabular}

Table 3: Biomicroscopy grading scores using the Efron grading scale (mean \pm standard deviation).

\subsection{Assessment of ocular surface fluorescence}

Pre-clinical laboratory testing confirmed that the series of seven photographs (10 micron thick F-WGA solution trapped between slides) captured using the custom imaging system, did not result in a significant change in the intensity of the F-WGA fluorescence (a difference in fluorescence of less than one pixel unit between all seven images - the equivalent of less than $2 \%$ of the mean fluorescent signal for these images).

Figure 1 contrasts F-WGA associated ocular surface fluorescence captured with a typical slit lamp imaging system and with the custom imaging system used in this clinical study. This highlights the sensitivity of the system to very low light levels and the even illumination provided by the custom imaging system. Figure 2 shows a typical sequence of images of F-WGA fluorescence across the bulbar and tarsal regions of the ocular surface at the five minute post-F-WGA application time point. The linear mixed model found a significant effect for time point $(\mathrm{F}=804.5, \mathrm{p}<0.0001)$ and ocular surface region $(\mathrm{F}=80.8, \mathrm{p}<0.0001)$, a borderline effect for participant group $(\mathrm{F}=3.1, \mathrm{p}=0.065)$ and no effect for age $(\mathrm{F}=1.1, \mathrm{p}=0.50)$. Given that a significant difference was observed between the two time points (5-minutes and 30-minutes post F-WGA application), a separate statistical model was undertaken for each time point.
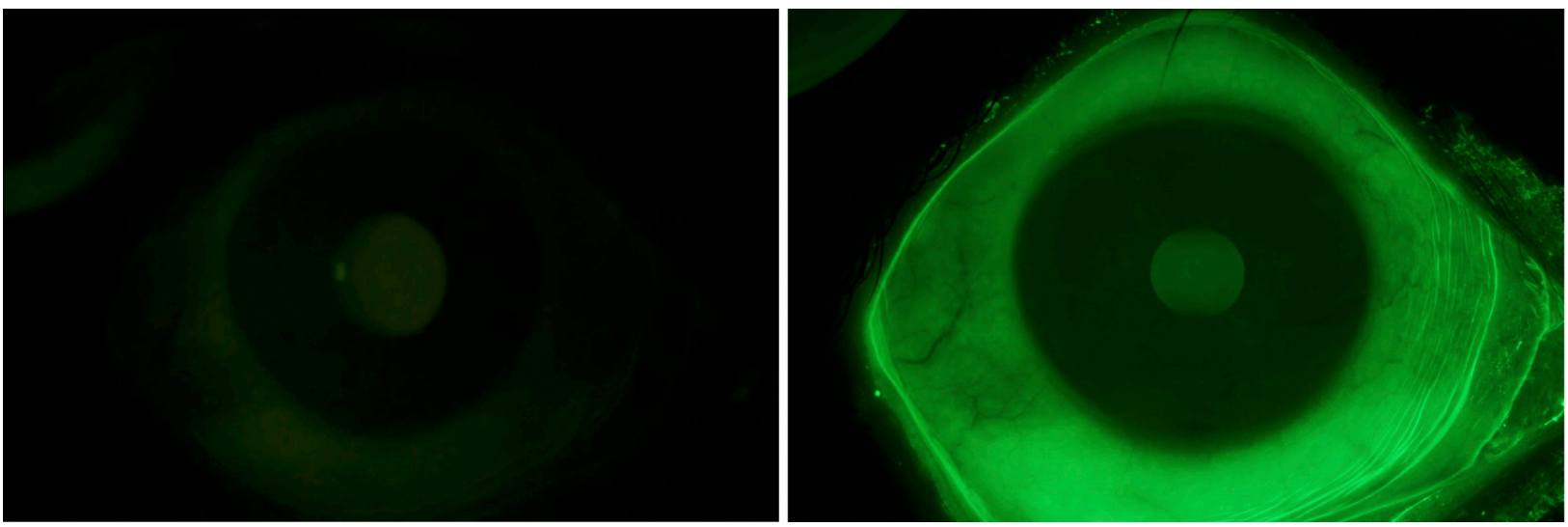

Figure 1: Comparison of a standard slit lamp imaging system (left) and the custom imaging system (right) for visualising F-WGA fluorescence 5 minutes after application of F-WGA solution.

\subsection{Ocular surface fluorescence 5-minutes post application of F-WGA}

Figure 3 highlights the degree of background-subtracted fluorescence measured at the seven ocular surface regions for the three different participant groups, at the five minute imaging time point. The linear mixed model found a significant effect for ocular surface region $(\mathrm{F}=64.7, \mathrm{p}<0.0001)$, a borderline effect for 

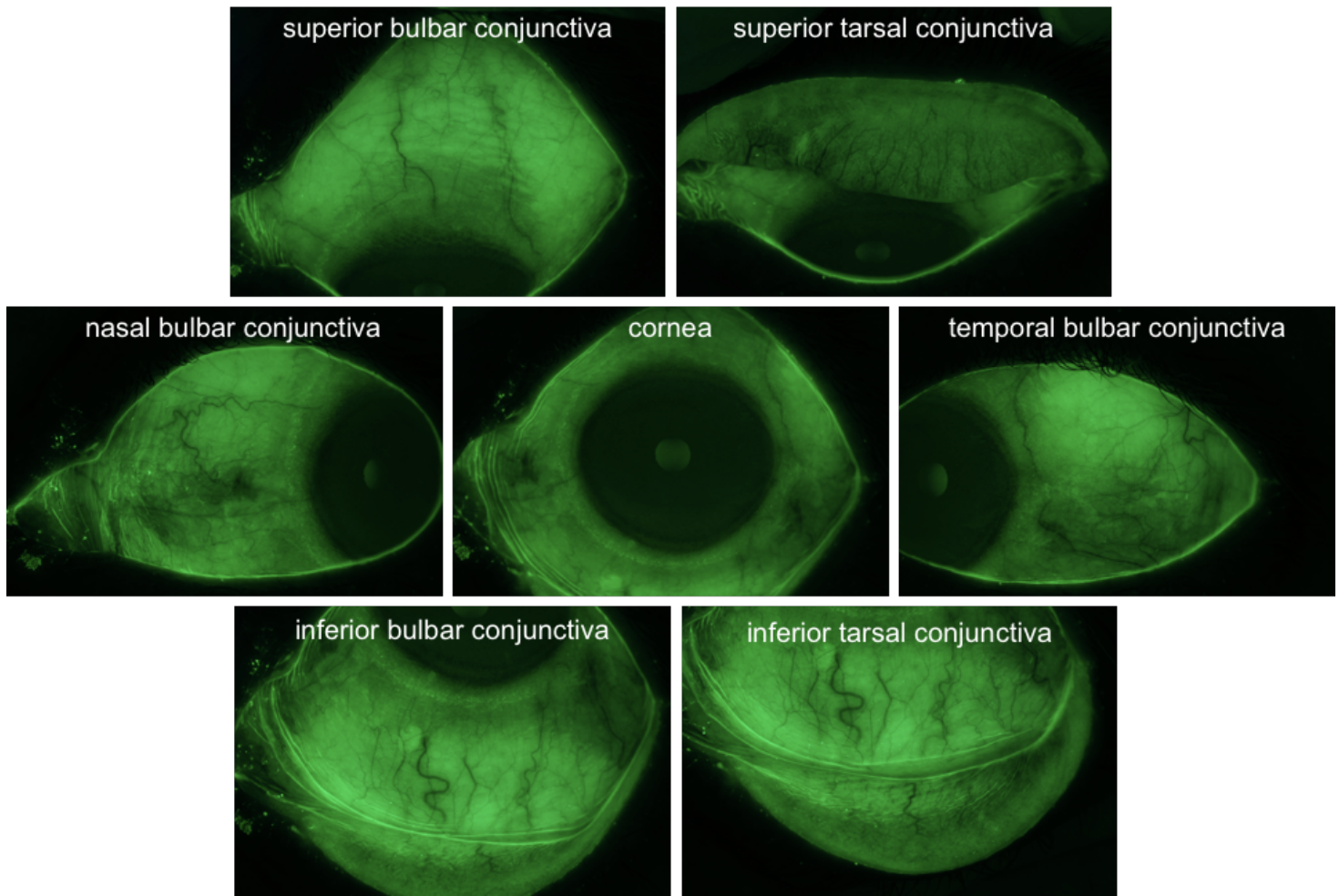

Figure 2: Typical images of F-WGA staining across the bulbar and tarsal regions of the ocular surface, captured using the custom imaging system. 
participant group $(\mathrm{F}=3.1, \mathrm{p}=0.07)$, whilst no effect was evident for age $(\mathrm{F}=1.0, \mathrm{p}=0.50)$. A Tukey posthoc analysis indicated that the greatest ocular surface fluorescence was observed on the nasal conjunctiva, followed by a group containing superior, temporal and inferior conjunctiva, another group containing both lid regions and the lowest level of fluorescence for the cornea. Given the marked difference in fluorescence between the ocular surface regions, a separate statistical model was run for each region. In these models, participant group was found to be a significant effect for the corneal region $(\mathrm{F}=4.2, \mathrm{p}=0.03)$, and the temporal conjunctiva $(\mathrm{F}=3.9, \mathrm{p}=0.04)$, with the participant group approaching significance for the upper tarsal conjunctiva $(\mathrm{F}=2.9, \mathrm{p}=0.08)$. A Tukey post-hoc analysis showed that the significant difference was between the asymptomatic lens wearing group and the non-lens wearing group, for both the corneal and temporal conjunctiva regions.

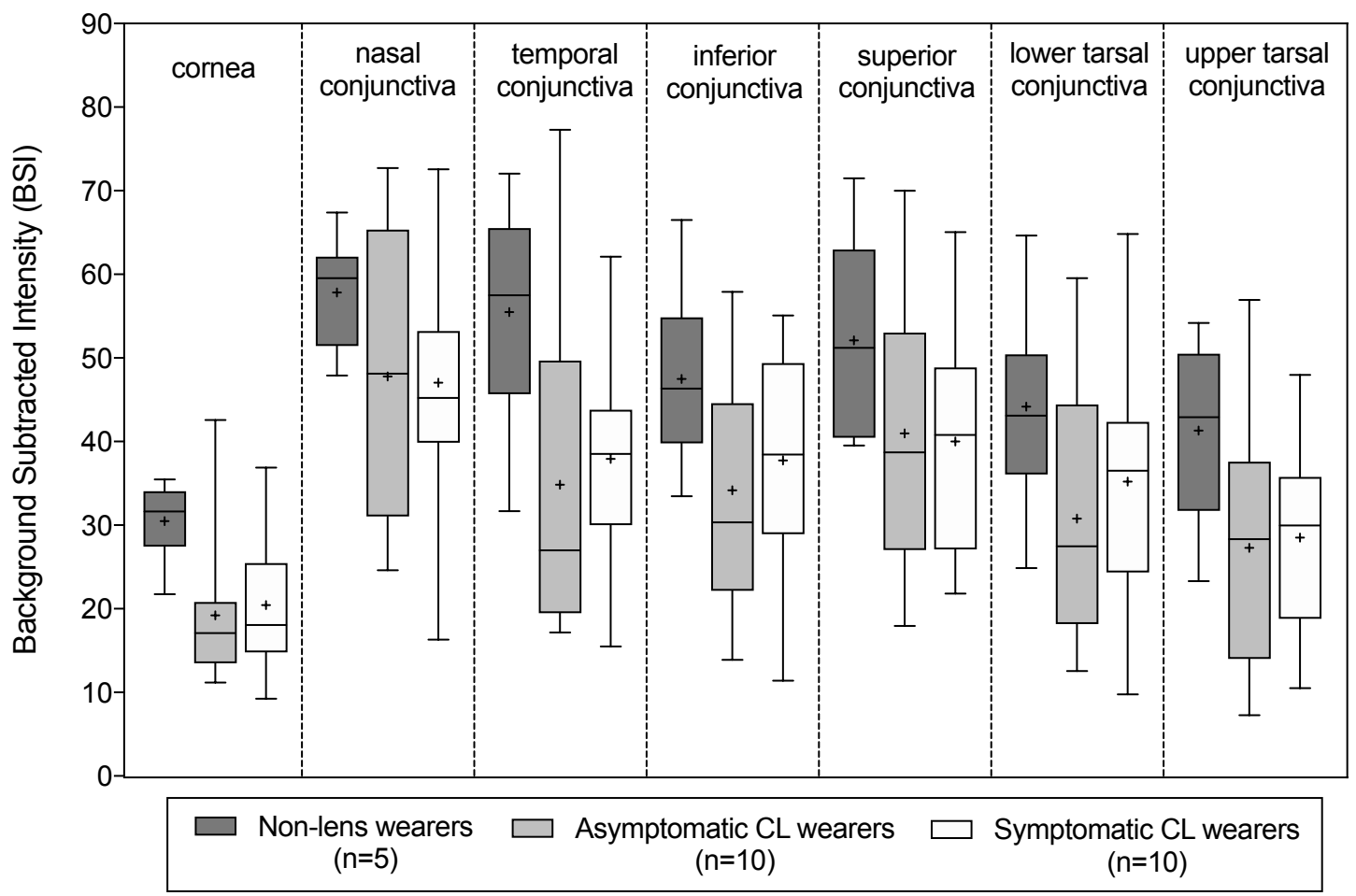

Figure 3: BSI of the seven ocular surface regions for the three participant groups at the 5-minute post-F-WGA application time point (a cross represents the mean, a horizontal line the median, the rectangles represent the 25 th and 75 th percentiles and the whiskers represent the 10th and 90th percentiles).

\subsection{Ocular surface fluorescence 30-minutes post application of F-WGA}

Figure 4 highlights the degree of background-subtracted fluorescence measured at the seven ocular surface regions for the three different participant groups, at the 30-minute time point. The linear mixed model found a significant effect for ocular surface region $(\mathrm{F}=28.6, \mathrm{p}<0.0001)$, an effect approaching significance for participant group $(\mathrm{F}=2.9, \mathrm{p}=0.07)$ and no significant effect for age $(\mathrm{F}=0.95, \mathrm{p}=0.56)$. A Tukey posthoc analysis was performed on the ocular surface region effect, indicating that the greatest ocular surface fluorescence was observed on the nasal conjunctiva, followed by a statistically similar grouping of superior conjunctiva, inferior conjunctiva and lower lid, with another statistically similar group (with a lower degree of fluorescence) including the upper lid and temporal conjunctiva regions and the group with the lowest level of fluorescence contained the cornea. Given the marked difference in fluorescence between the ocular surface regions, a separate statistical model was run for each region. In these models, participant group was found to be a significant effect for the cornea $(\mathrm{F}=5.5, \mathrm{p}=0.01)$ and approaching statistical significance for the lower 
tarsal conjunctiva $(\mathrm{F}=2.7, \mathrm{p}=0.09)$ and temporal conjunctiva $(\mathrm{F}=3.2, \mathrm{p}=0.06)$. A Tukey post-hoc analysis demonstrated that the statistically significant difference observed for the corneal region was between the non-lens wearing group and both the symptomatic and asymptomatic contact lens wearing groups.

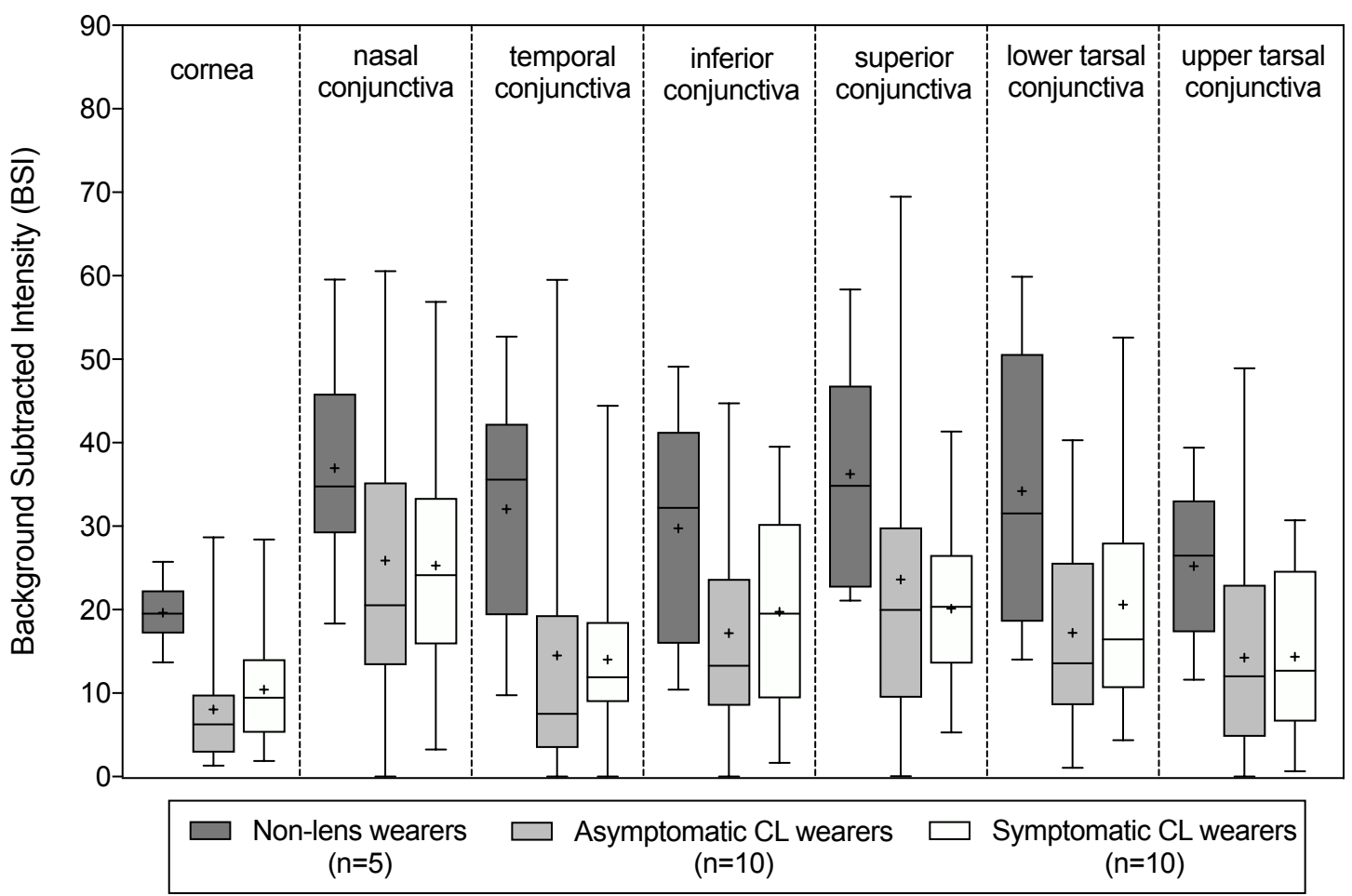

Figure 4: BSI of the seven ocular surface regions for the three participant groups at the 30-minute post-F-WGA application time point (a cross represent the mean, a horizontal line the median, the rectangles represent the 25th and 75th percentiles and the whiskers represent the 10th and 90th percentiles)

\subsection{Ocular surface fluorescence in the lid wiper region}

Assessment of F-WGA-associated fluorescence of the tarsal conjunctiva reported in sections 3.4 and 3.5 was based on an average of the pixel intensity across the entire everted lid region. Further image analysis was also undertaken to characterise fluorescence specific to the lid wiper region (using the methodology described in Section 2.1). Figure 5 shows the fluorescence profile across the lid wiper and tarsal conjunctiva, for the three participant groups. The fluorescence intensity profile for the asymptomatic group generally demonstrated a similar pattern to the no-lens wear group, whereas the symptomatic group appeared to exhibit decreased fluorescence in the lid wiper region. To quantify this apparent reduction in F-WGA associated fluorescence in the lid wiper region, the difference in fluorescence between the the lid wiper region (a region of the tarsal conjunctiva less than $0.6 \mathrm{~mm}$ from the line of Marx) and a reference region on the everted eyelid (a region of the the tarsal conjunctiva at least $1 \mathrm{~mm}$ away from the line of Marx) was calculated. Figure 6 shows the difference in fluorescence between these two regions, with the asymptomatic contact lens wearers and non-lens wearers exhibiting similar or greater levels of fluorescence in the lid wiper region, whereas for the symptomatic lens wearers the fluorescence was consistently lower in the lid wiper region ( $95 \%$ confidence intervals not crossing zero for all four lid / study time point combinations). A linear mixed model for difference in F-WGA associated fluorescence between the lid wiper region and the reference tarsal conjunctiva region, demonstrated (i) a difference between the participant groups $(\mathrm{F}=4.1, \mathrm{P}=0.03)$, with a post-hoc analysis indicating a relative reduction in lid wiper fluorescence for the symptomatic contact lens participants, in comparison with the asymptomatic participants, and (ii) a difference between the eyelids 
$(\mathrm{F}=16.6, \mathrm{P}<0.0001)$, with the upper lid wiper region displaying less fluorescence than the reference region, whereas the lower lid showed greater relative fluorescence in this region. The difference in fluorescence between these two tarsal regions did not appear to be influenced by the imaging time point $(\mathrm{F}=2.2, \mathrm{p}=0.14)$, eye $(\mathrm{F}=1.2, \mathrm{p}=0.26)$, age $(\mathrm{F}=0.2, \mathrm{p}=0.69)$ or by any of the interaction terms evaluated ( $\mathrm{p}>0.05$ in all cases).

\subsection{Ocular surface fluorescence in the corneal limbal region}

Imaging of the bulbar conjunctiva following application of F-WGA highlighted the presence of an annular band of hypofluoresence (Figure 7a) or hyperfluoresence (Figure 7b), in the majority of contact lens wearing participants (hypofluoresence in $60 \%$ of lens wearering eyes and hyperfluoresence in $30 \%$ of lens wearering eyes). These observations were not apparent in the non-lens wearing participants. To better characterise these observations, an image analysis algorithm was applied to the primary gaze study images (using the methodology described in Section 2.1). Comparison of the average radial profile (Figure 8), showed a greater level of fluorescence observed for the non-lens wearing participant group, in comparison with the two lens-wearing groups in the corneal and limbal regions. Figure 8 highlights that the annular band of negative staining or hyper-fluorescence was not observed on the bulbar conjunctiva of the non-lens wearing participants (e.g. a smooth transition in fluorescence across the bulbar conjunctiva). In contrast, both the symptomatic and asymptomatic contact lens participant groups showed an apparent notch in fluorescence around $1.2 \mathrm{~mm}$ outside of the limbus. The location and depth of this notch was broadly similar between the two participant groups and appeared to be induced by the contact lens edge. Inspection of the individual participant radial cross-section plots, showed that the circumferential band of negative staining was a far more typical observation than the circumferential band of hyperfluoresence. Inspection of the individual participant radial cross-section plots was also undertaken to investigate the influence of lens type (lens material / modality) on this observation, but no obvious pattern was evident.

\section{Discussion}

The custom imaging system was able to detect the low levels of fluorescence emitted by F-WGA, even at the 30 minute post-application time point. The images captured demonstrate the ability of the system to avoid motion blur and photobleaching of the fluorophore, whilst providing increased depth of field, even illumination and consistent image capture. It was apparent that the application of F-WGA did not appear to influence ocular surface physiology or visual acuity significantly; however, some study participants did report a mild transient foreign body sensation, which appeared to be most apparent in the non-lens wearing participants. As the suspension was micro-filtered during preparation and centrifuged prior to application, this sensation is unlikely to be associated with F-WGA aggregation (supported by a lack of observable aggregation following application) and may relate to the interaction of the dye with the ocular surface mucins or be due to a difference in the $\mathrm{pH}$ or osmolarity of the solution compared with the tear film of the participants. The installation of F-WGA solution onto the ocular surface resulted in a fluorescent pre-corneal tear film and menisci, similar in appearance to that observed after application of sodium fluorescein (albeit with substantially weaker fluorescence). As the F-WGA solution was gradually cleared from the tear film, it was apparent that some of the F-WGA had bound to the bulbar and tarsal mucosal surfaces, with a clear demarcation at the line of Marx, as would be expected given the known bindings characteristics of F-WGA $[5,6,10]$. WGA has been used extensively as a marker for the epithelial glycocaylx [19-21] and has been shown to be highly specific to N-acetyl-D-glucosamine and sialic acid [22-24]. It has be used widely in ex vivo imaging of corneal tissue to visualise the ocular surface glycocaylx $[21,25,26]$ and in tear film and worn contact lens analysis, where it acts as a marker of mucins [3, 27]. More recent clinical use of F-WGA has highlighted that a mucolytic agent (5\% N-acetylcysteine) reduced F-WGA binding [10], whilst two topical pharmacological agents that promote mucin production (2\% rebamipide - a mucin secretagogue and $3 \%$ diquafosol tetrasodium - a P2Y2 receptor agonist) have both been shown to increase F-WGA binding, with the rebamipide study also detecting an increased sialic acid concentration in the tear film [8, 28]. The literature is therefore clear that F-WGA is primarily acting as a specific marker for ocular surface mucins, with minimal non-specific binding anticipated. This is supported by the observation of fluorescent strands 
5 minute timepoint: Lower lid

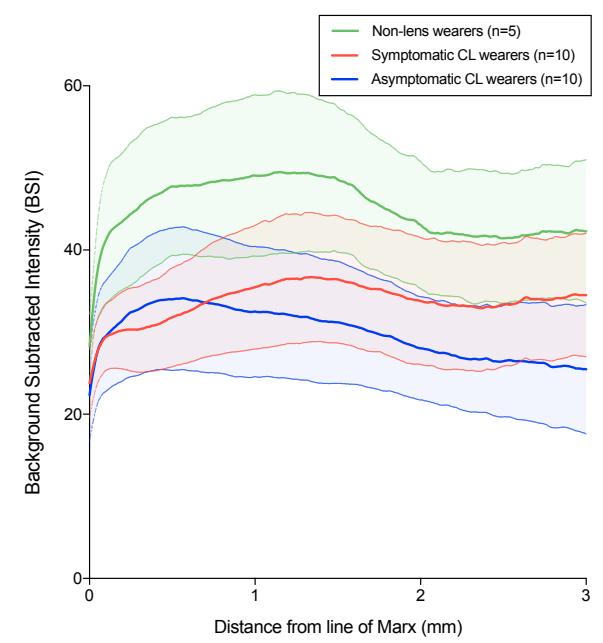

30 minute timepoint: Lower lid

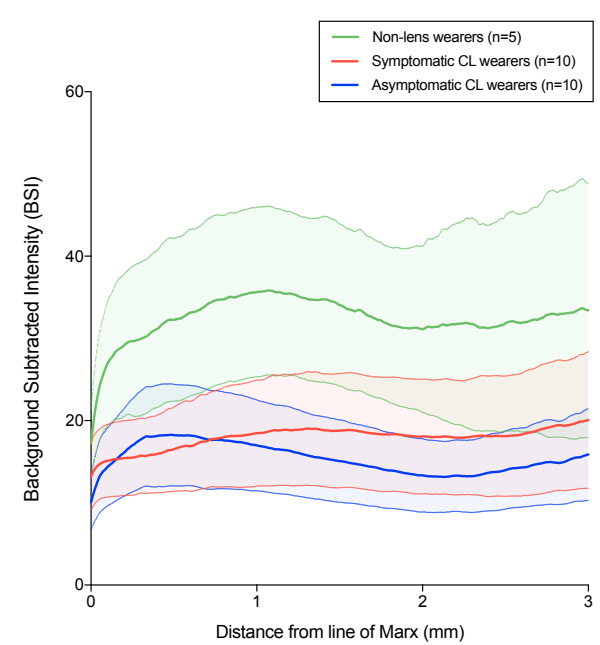

5 minute timepoint: Upper lid

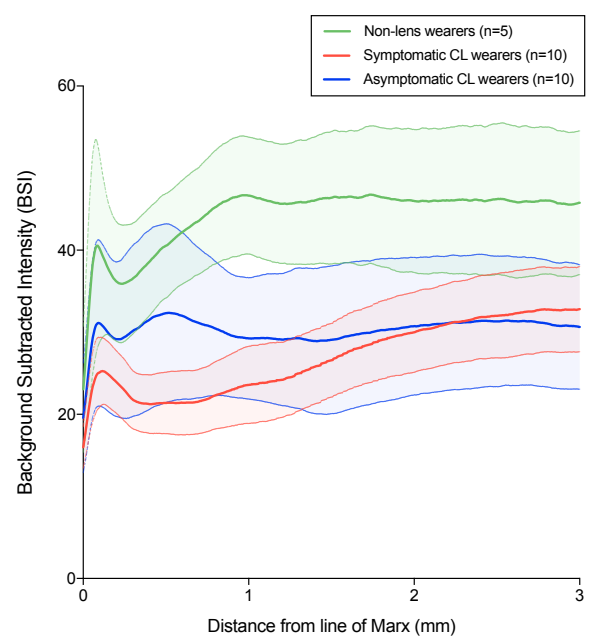

30 minute timepoint: Upper lid

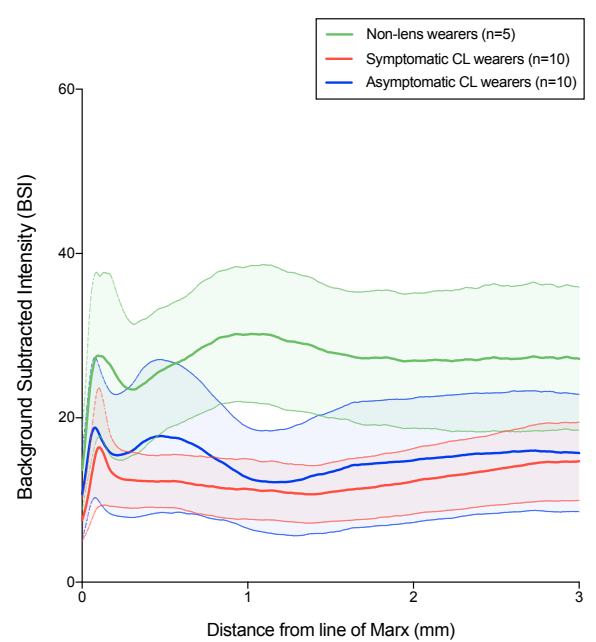

Figure 5: The intensity of F-WGA fluorescence across the lid wiper and tarsal conjunctiva, for the upper and lower lids and the 5-minute and 30-minute time points, for the three participant groups (the solid line indicates the mean intensity profile and the shaded regions highlight the $95 \%$ confidence intervals). 

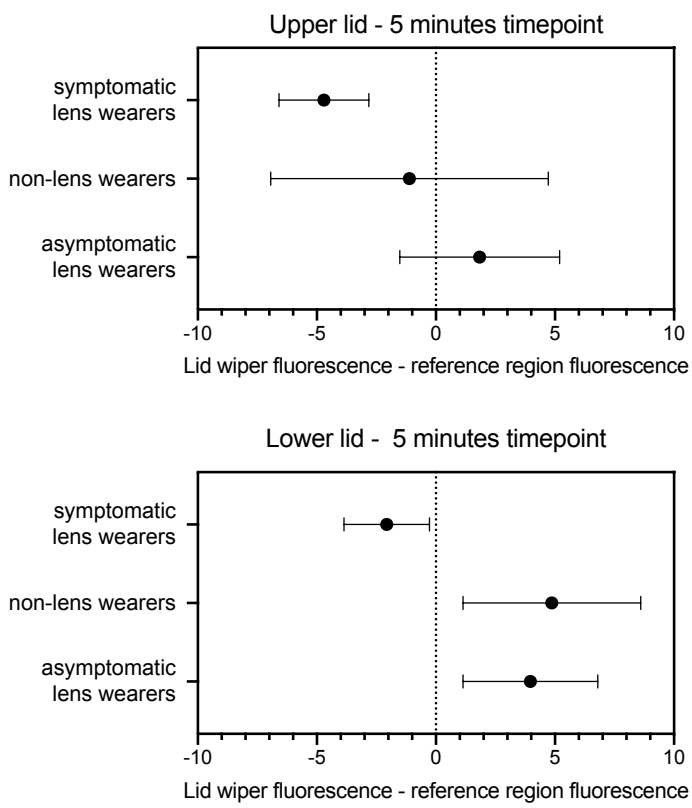
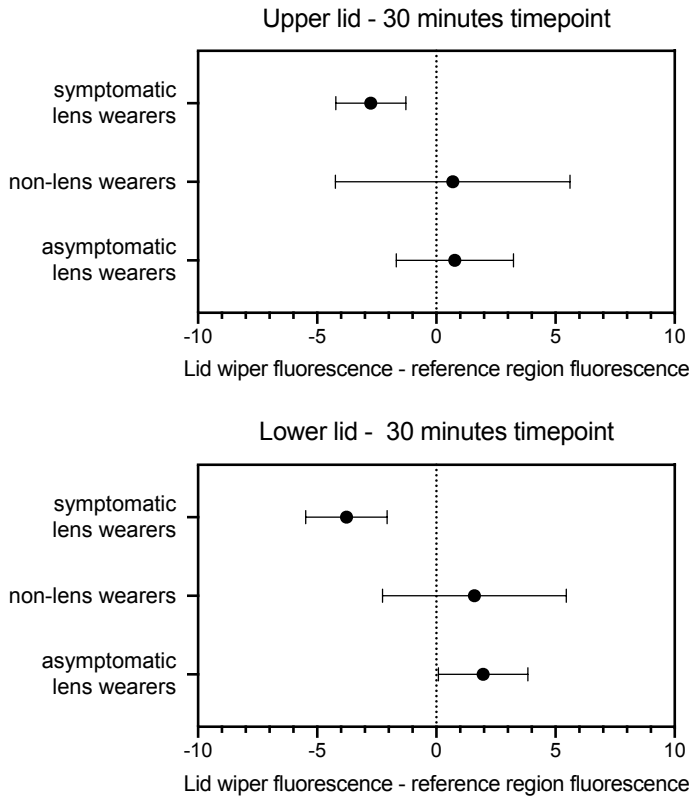

Figure 6: The difference in mean background-subtract fluorescence between the lid wiper region (up to $0.6 \mathrm{~mm}$ from line of Marx) and a reference region of the tarsal conjunctiva ( $>1 \mathrm{~mm}$ from the line of Marx) for the four imaging time point / eyelid combinations. Error bars represent the $95 \%$ confidence intervals.
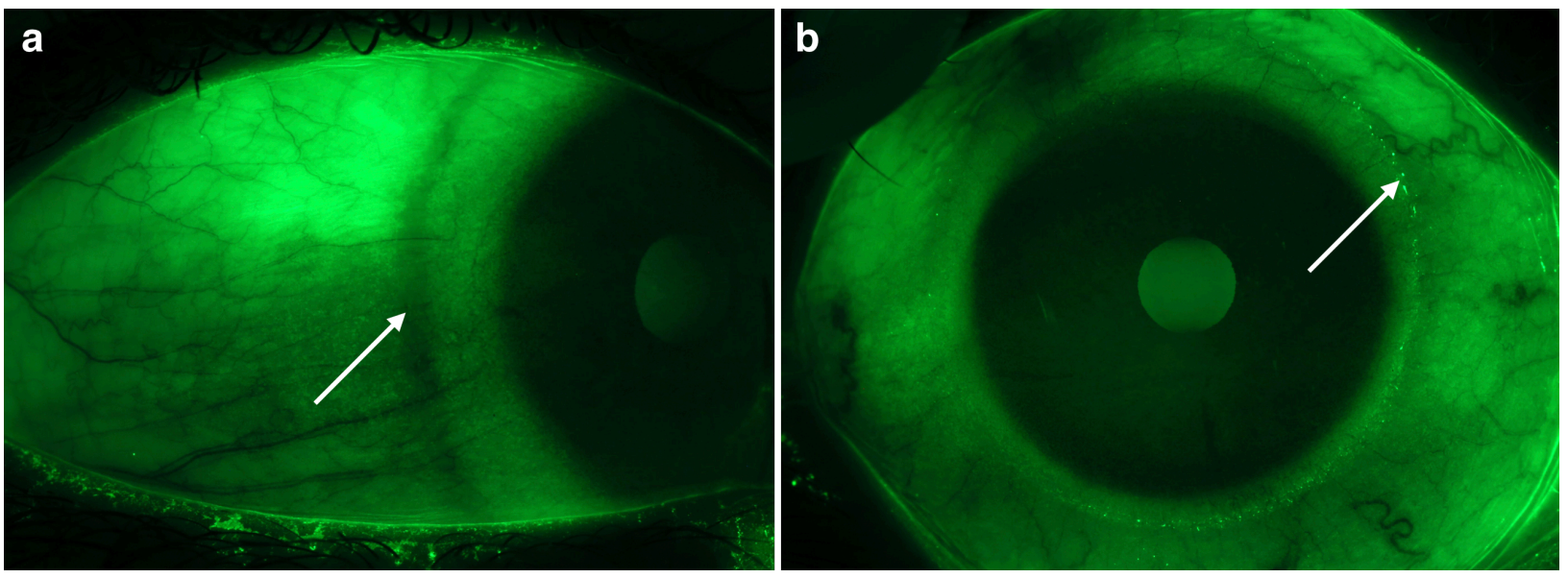

Figure 7: Typical images of annular negative staining (a) and hyperfluorescence (b) on the bulbar conjunctiva of contact lens wearing participants. 


\section{5-minute timepoint}

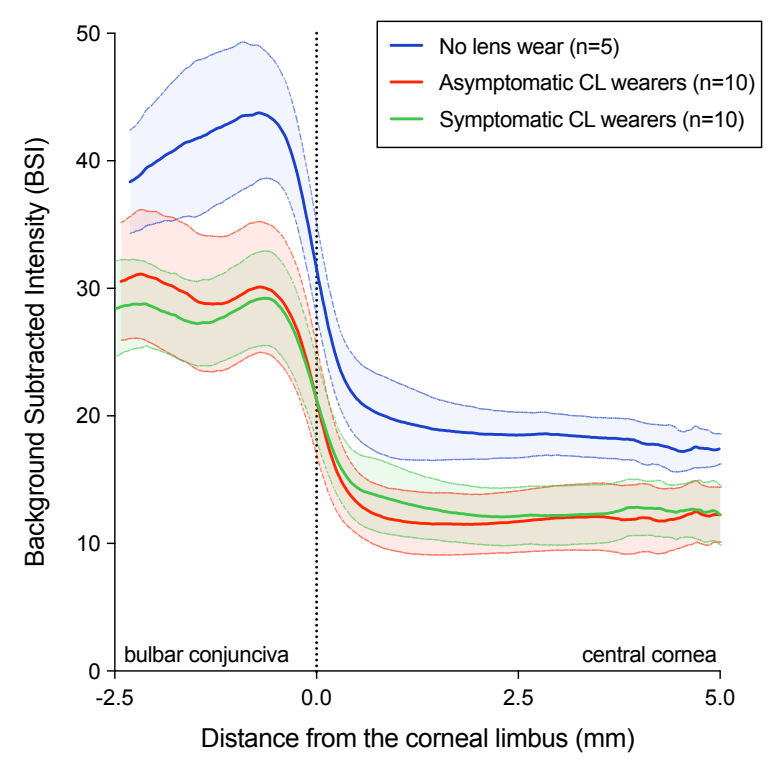

\section{0-minute timepoint}

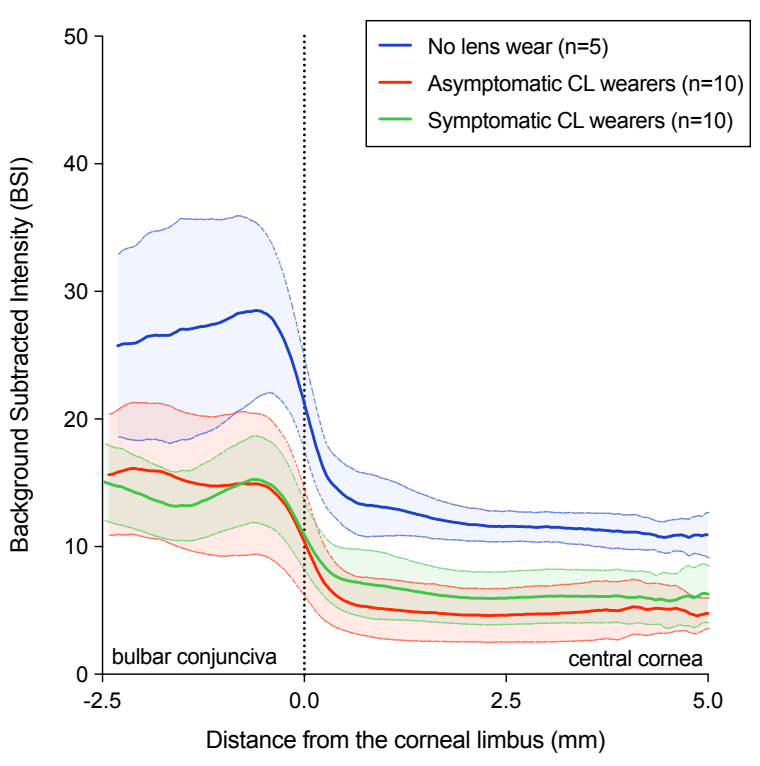

Figure 8: A fluorescence intensity profile of the cornea / bulbar conjunctiva based on averaging radial scans centered on the cornea.

in this clinical study, which aggregate towards the lacrimal caruncle, in a manner classic of mucus stands. In addition, the distribution of fluorescence observed in this study is typical of that described in the literature for mucin and goblet cell distribution across the ocular surface [29-31]. Given that unbound F-WGA is rapidly cleared from the tear film, as is typical following application of ocular surface dyes [32, 33], the principle source of ocular surface fluorescence at both time points is associated with mucin-bound F-WGA. This is supported by the clinical observation that fluorescent features are static on the ocular surface (i.e. the features do not move/change with the blink) and that there is minimal fluorescence of the tear film meniscus.

At the 5-minute post-application time point, the distribution of bound F-WGA across the bulbar and tarsal surfaces appeared relatively uniform. Statistical analysis highlighted that fluorescence differed between the seven ocular surface regions and was greatest across the bulbar conjunctiva and lowest on the cornea. This appears to suggest a greater mucin density on the bulbar conjunctiva, followed by the tarsal conjunctiva, with the lowest concentration at the cornea; although other factors may also influence F-WGA binding. These observations are in agreement with previous reports of greater F-WGA fluorescence on the bulbar conjunctiva in comparison with the cornea [10]. This previous work relied on an analogue photo-multiplier tube to detect the fluorescence over a wide area (typically a $10 \mathrm{~mm}$ region), unlike the system describe here which is able to capture F-WGA distribution with high spatial resolution allowing a far greater degree of information to be captured.

Over the 30-minute study period, it was apparent that the degree of ocular surface florescence gradually reduced over time. Although the initial reduction in fluorescence was likely to be associated with clearance of F-WGA solution from the tear film, the reduction in fluorescence later in this period appeared to be associated with partial clearance and aggregation of the surface-bound F-WGA. Typically this aggregation occurred on a microscopic scale, with the aggregates then cleared from the tear film via the puncta, but for many participants (around $60 \%$ of contact lens wearers and $90 \%$ of non-lens wearers) fluorescent mucous strands were also clearly visible (Figure 9), which tended to aggregate towards the lacrimal caruncle. It is interesting to note that these strands were not obvious in white light examination of the ocular surface. Further work is required to understand if these observations are associated with the normal turnover of the 
ocular surface mucins, or whether application of the F-WGA solution influences this turnover process.
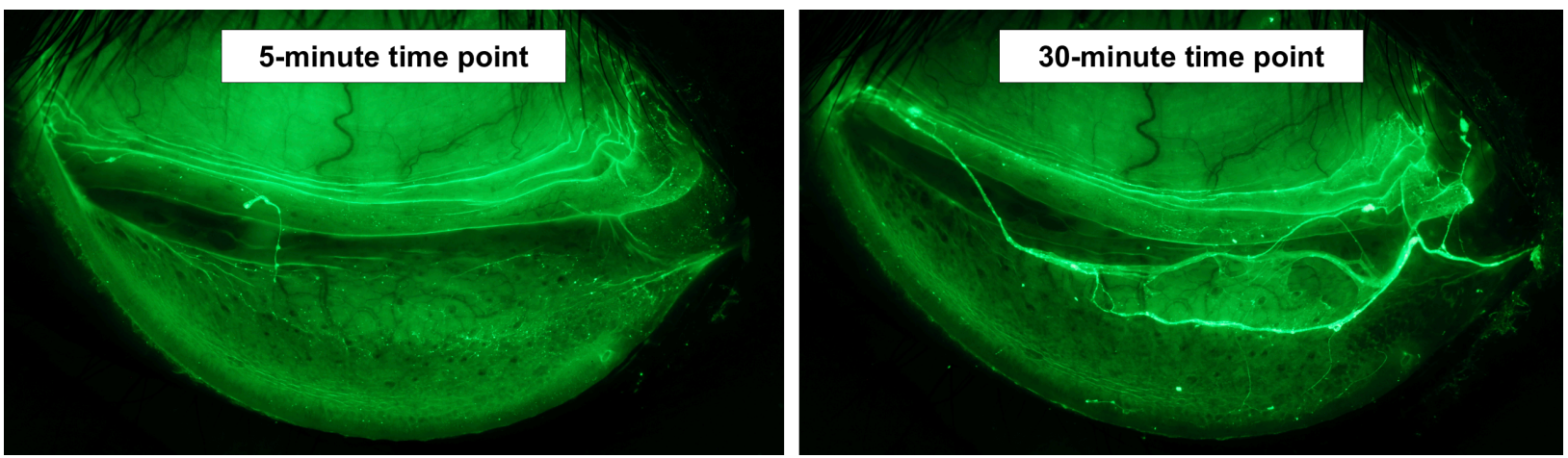

Figure 9: Development of mucus strands through the 30-minute imaging period.

Ocular surface fluorescence was observed to diminish between the two study imaging time points, with the ocular surfaces with greater fluorescence (the bulbar conjunctival surfaces) typically showing the greatest reduction in fluorescence. Possible reasons for this may include (i) clearance of any F-WGA solution pooling in these regions at the 5-minute-time point, (ii) a difference in shear forces experienced by the different ocular tissues during blinking and (iii) a difference in the degree of F-WGA binding for the different ocular surface regions. This observation of reducing fluorescence with time is a new finding, with previous clinical studies using F-WGA solutions [10] finding a consistent level of fluorescence over a 30-minute period. These differences are likely due to the increased sensitivity of our custom imaging system, allowing this change in fluorescence to be more accurately monitored, although other factors including the study participant population (predominately white European participants in this study vs. East Asian participants) or due to differences in the subtleties of the imaging methodology or F-WGA preparation. Although the results observed at the 5-minute and 30-minute time points appear to provide similar information, the 5-minute time point appears to capture the initial distribution of the ocular surface mucins, whereas the 30-minute time point appears to give an insight into mucin turnover at the ocular surface. Assessment of mucin distribution and turnover may help to identify areas where excessive interaction between the ocular surfaces or between an ocular surface and a contact lens surface results in mucin depletion. Future studies should consider characterising the time course of F-WGA binding and clearance to better understand these processes. It should be noted that although there is substantial evidence that F-WGA acts a marker for mucins at the ocular surface, it is not known whether F-WGA is selectively binding transmembrane mucins (e.g. MUC1/MUC4/MUC16 in the epithelial glycocalyx), gel forming mucins (e.g. MUC5AC) or a combination of both. Future studies should look to characterise the binding specificity of WGA to a range of ocular surface mucins, to further our understanding of what is being visualised by this technique.

Although F-WGA ocular surface florescence was generally uniform, on two occasions a region of negative staining was observed on the cornea (Figure 10). The cause of this negative staining is unclear, but may be associated with contact lens wear or the lens removal process, as no such observations were made on the non-lens wearing participants. It is interesting to note that such episodes of negative staining did not result in sodium fluorescein staining in these regions, indicating that an absence of surface mucins is insufficient in itself to result in sodium fluorescein uptake into the surface epithelial cells - an important observation.

Comparison of ocular surface fluorescence between the three participant groups revealed consistent differences. Whilst the asymptomatic and symptomatic contact lens wearing groups appeared to exhibit similar F-WGA binding across the seven ocular surface regions, the fluorescence observed for the non-lens wearing group was consistently higher. This suggests that contact lens wear negatively impact mucin density across these surfaces and/or influences their structure sufficiently to alter the degree to F-WGA binding. This observation appears consistent across all seven ocular surface regions and is in agreement with that reported in the literature [9]. In addition to analysis of the seven ocular surface regions, more specific analysis was undertaken on two specific regions where contact lens wear is known to interact with the ocular surface 


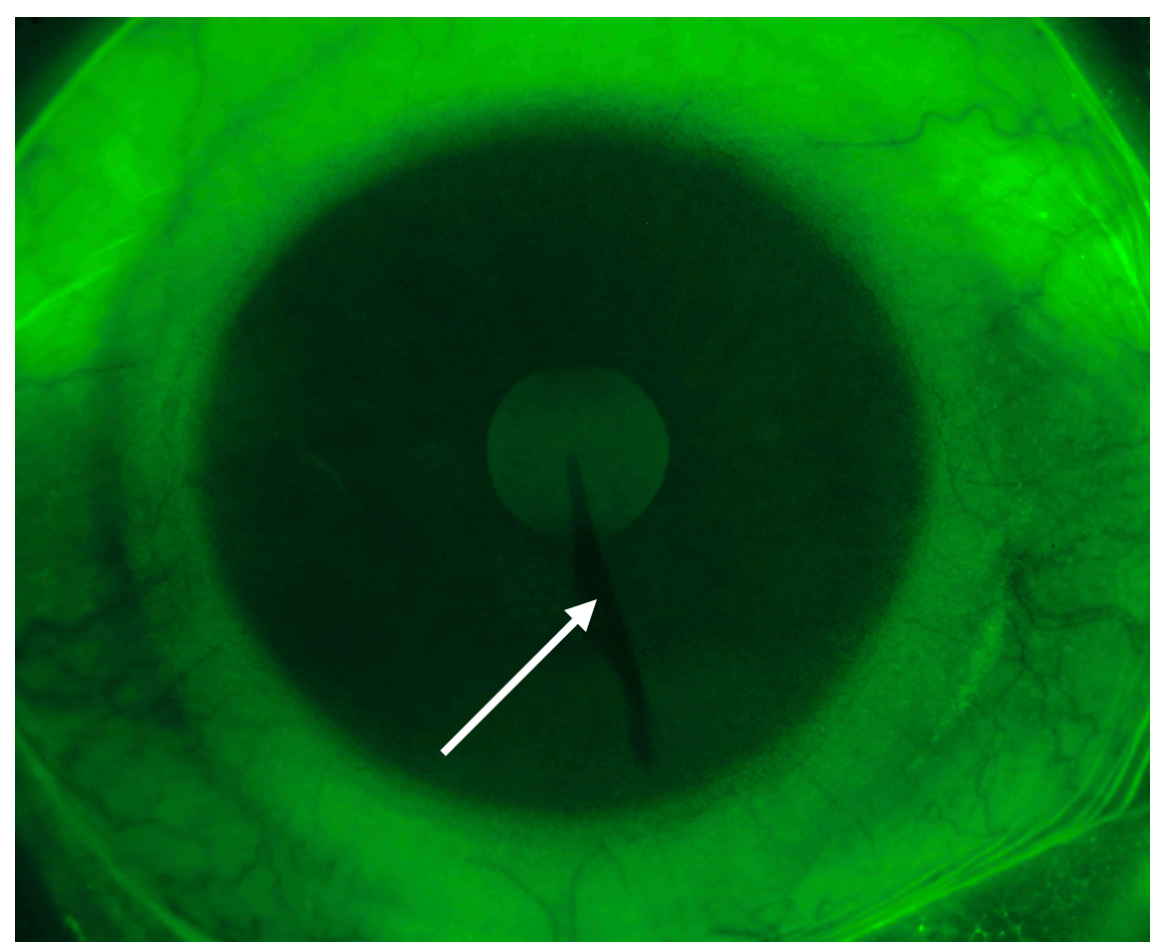

Figure 10: An example photograph highlighting a vertical streak of negative staining (white arrow) across the cornea.

[11, 34-37]. The bulbar conjunctiva underlying the contact lens edge and the lid wiper region of the tarsal conjunctiva both underwent custom image analysis in order to compare the degree of fluorescence across the three participant groups. For the two lens-wearing participant groups, the bulbar conjunctiva underlying the lens edge demonstrated a reduction in F-WGA fluorescence. This reduction in fluorescence is thought to be associated with the lens edge interacting excessively with the bulbar conjunctiva and reducing the abundance of ocular surface mucins in this region. This is in agreement with the literature, where increased fluorescence is observed in this region following application of sodium fluorescein, likely indicating cellular disturbance in these regions. An alternative explanation for the negative staining could be flurophore quenching [38], as has been reported clinically with sodium fluorescein [39], however due to the low fluorophore concentration and the consistent hypofluorescence in this circumlimbal region over the two time points (whilst there was a significant reduction in overall fluorescence), this likely indicates genuine hypofluorescence in this region, rather than flurophore quenching.

Although the level of F-WGA fluorescence in this region was lower for the contact lens wearing groups, there were no obvious differences between the symptomatic and asymptomatic groups. This observation suggests that contact lens edge / conjunctival surface interaction is unlikely to be a primary driver of discomfort in the symptomatic contact lens wearing population, in agreement with previously work finding no association between lens-edge related conjunctival staining and comfort [18, 40-42]. In contrast, the level of F-WGA fluorescence observed in the lid wiper region was not only substantially reduced for the contact lens wearers in comparison with non-lens wearers, but also appeared to differ between the asymptomatic and symptomatic groups. This difference was more marked when the relative change in fluorescence across the lid wiper region was considered (to account for differences in F-WGA fluorescence intensity between participants). These observations appear to be in agreement with the literature, where symptomatic patients are reported to experience greater levels of sodium fluorescein and lissamine green staining in this region $[3,36]$. Korb et al. [36], have suggested that an increased interaction occurs between the contact lens and the lid wiper region in symptomatic lens wearers, resulting in a reduction in the abundance of ocular surface mucins in the lid wiper region. Such an observation after a relatively short period of lens wear (on average 6 
hours in this study), suggests that over more typical lens wearing periods (circa 12 hours of lens wear for the participants in this study), the ocular surface mucins could be further diminished, resulting in increasing frictional interaction at this interface. Such an interaction has the potential to result in a reduction in subjective comfort, either by direct contact with the lid wiper tissue (especially given the high sensitivity of this tissue [43]) or by increased contact lens motion resulting in associated irritation of the corneal/bulbar conjunctival tissue. Future studies should assess F-WGA fluorescence in the lid wiper region over more prolonged periods of lens wear, to understand mucin distribution changes later in the lens wearing cycle. In addition, future work could also look at the co-localisation of ophthalmic dyes across the ocular surface as the information they provide is likely to be complementary.

As this study was designed to assess F-WGA staining following a period of habitual lens wear, the participants presented with a range of lens types, modalities and duration of lens wear that day. This approach provided a good overview of the impact contact lens wear can have on the ocular surface mucins, but it made distinguishing lens-related factors which influence mucin distribution more challenging. Future studies should therefore consider a more systematic approach to examining the impact of different lens designs and materials on ocular surface mucins (e.g. cross-over study design with washout periods), to improve our understanding of the contact lens characteristics that could be enhanced to minimise interaction with the ocular surface.

In addition to its application in investigating the aetiology of contact lens discomfort and in developing contact lens materials and designs that minimise their interaction with the ocular environment, there are a number of other fields where F-WGA may be a useful research and/or clinical tool. Given the short residency time of ophthalmic drops on the ocular surface, there is significant interest in the development of mucoadhesive pharmacological agents to maximise their therapeutic effect and minimising side effect [44, 45]. The use of F-WGA is likely to be a key tool in understanding how ocular surface mucin structure varies within the population and how this might impact on such a drug delivery mechanism. In addition, the distribution of ocular surface mucins is thought to be impacted in dry eye disease, keratoconus, ocular surface disease and post-ophthalmic surgery [6, 46-48]. F-WGA is therefore likely to be a key tool in visualising ocular surface mucin distribution and monitoring treatment strategies.

From a clinical perspective, this study gives eye care practitioners (ECP) an insight into the normal distribution and turnover of ocular surface mucins and how contact lens wear reduces their abundance, particularly over the cornea. Such changes have been reported to influence ocular surface wettability [9], are associated with compromised ocular surface friction [49-51] and may play a role in the increased risk of infection observed with contact lens wear [52, 53], although further work is needed to understand this relationship. The work also highlights that the interaction of the lens edge with the ocular surface does not appear to negatively influence ocular comfort, however increased interaction in the lid wiper region does appear to be associated with greater symptomotology. Clinicians should therefore look to optimise a patient's tear film quality and quantity, along with the careful selection of contact lens material and design (particularly in relation to tear film wettability and lens surface friction) to minimise physical insult and biological wear in this region. In addition, this work also opens up the possibility of a future clinical ophthalmic dye which would allow the ECP to grade the quality of ocular surface mucins in a more quantitative manner than the binary-type of staining that is observe with existing ophthalmic dyes, such as sodium fluorescein and lissamine green [54].

\section{Conclusions}

The use of F-WGA as a clinical ophthalmic marker has provided useful insights into the distribution of mucins across the ocular surface. It has highlighted that mucin concentration appears greatest on the bulbar conjunctiva and lowest on the cornea. Contact lens wear has been shown to reduce F-WGA ocular surface binding across the cornea, tarsal conjunctiva and bulbar conjunctiva, which may be important in a range of clinical issues including comfort, vision and inflammation and/or infection. Across the bulbar conjunctiva and the cornea, no significant differences in F-WGA binding were observed between symptomatic and asymptomatic contact lens wearers in this study. However, specific image analysis on the lid wiper regions demonstrated a difference in F-WGA distribution between symptomatic and asymptomatic contact 
lens wearers, with a reduced F-WGA fluorescence in the lid wiper region for the symptomatic contact lens wearers. This study therefore demonstrates the utility of F-WGA as an ophthalmic dye and details the methodology to allow its imaging in a clinical setting. Given the ability of F-WGA to disclose mucin distribution across the ocular surface it is likely to be a key clinical tool in furthering our understanding of (i) the aetiology of contact lens related discomfort, (ii) contact lens designs/materials which minimise their interaction with the ocular surface and (iii) dry eye disease and other ocular surface diseases.

\section{References}

[1] Gipson, I.K.. Distribution of mucins at the ocular surface. Exp Eye Res 2004;78(3):379-388.

[2] Govindarajan, B., Gipson, I.K.. Membrane-tethered mucins have multiple functions on the ocular surface. Exp Eye Res 2010;90(6):655-663.

[3] Berry, M., Pult, H., Purslow, C., Murphy, P.J.. Mucins and Ocular Signs in Symptomatic and Asymptomatic Contact Lens Wear. Optom Vis Sci 2008;85(10):E930-8.

[4] Mantelli, F., Argueso, P.. Functions of ocular surface mucins in health and disease. Curr Opin Allergy Clin Immunol 2008;8(5):477-483.

[5] Wright, C.S.. Structural comparison of the two distinct sugar binding sites in wheat germ agglutinin isolectin II. J Mol Biol 1984;178(1):91-104.

[6] Mencucci, R., Marini, M., Gheri, G., Vichi, D., Sarchielli, E., Bonaccini, L., et al. Lectin binding in normal, keratoconus and cross-linked human corneas. Acta Histochem 2011;113(3):308-316.

[7] Robin, J.B., Chan, R., Rao, N.A., Sharma, S., Srinivasan, M.. Fluorescein-conjugated lectin visualization of fungi and acanthamoebae in infectious keratitis. Ophthalmology 1989;96(8):1198-1202.

[8] Shigeyasu, C., Yamada, M., Akune, Y., Fukui, M.. Diquafosol for Soft Contact Lens Dryness: Clinical Evaluation and Tear Analysis. Optom Vis Sci 2016;93(8):973-978.

[9] Fukui, M., Yamada, M., Akune, Y., Shigeyasu, C., Tsubota, K.. Fluorophotometric Analysis of the Ocular Surface Glycocalyx in Soft Contact Lens Wearers. Curr Eye Res 2015;41(1):9-14.

[10] Mochizuki, H., Fukui, M., Hatou, S., Yamada, M., Tsubota, K.. Evaluation of ocular surface glycocalyx using lectin-conjugated fluorescein. Clin Ophthalmol 2010;4:925-930.

[11] Deng, Z., Wang, J., Jiang, H., Fadli, Z., Liu, C., Tan, J., et al. Lid Wiper Microvascular Responses as an Indicator of Contact Lens Discomfort. Am J Ophthalmol 2016;170:197-205.

[12] Pult, H., Purslow, C., Berry, M., Murphy, P.J.. Clinical Tests for Successful Contact Lens Wear: Relationship and Predictive Potential. Optom Vis Sci 2008;85(10):E924-E929.

[13] Korb, D.R., Herman, J.P., Greiner, J.V., Scaffidi, R.C., Finnemore, V.M., Exford, J.M., et al. Lid Wiper Epitheliopathy and Dry Eye Symptoms. Eye Contact Lens 2005;31(1):2-8.

[14] Korb, D.R., Herman, J.P., Blackie, C.A., Scaffidi, R.C., Greiner, J.V., Exford, J.M., et al. Prevalence of Lid Wiper Epitheliopathy in Subjects With Dry Eye Signs and Symptoms. Cornea 2010;29(4):377-383.

[15] Chalmers, R.L., Begley, C.G., Moody, K., Hickson-Curran, S.B.. Contact Lens Dry Eye Questionnaire-8 (CLDEQ-8) and Opinion of Contact Lens Performance. Optom Vis Sci 2012;89(10):1435-1442.

[16] Chalmers, R.L., Begley, C.G., Caffery, B.. Validation of the 5-Item Dry Eye Questionnaire (DEQ-5): Discrimination across self-assessed severity and aqueous tear deficient dry eye diagnoses. Cont Lens Anterior Eye 2010;33(2):55-60.

[17] Efron, N., Brennan, N.A., Morgan, P.B., Wilson, T.. Lid wiper epitheliopathy. Prog Retin Eye Res 2016;53:140-174.

[18] Morgan, P.B., Chamberlain, P., Moody, K., Maldonado-Codina, C.. Ocular physiology and comfort in neophyte subjects fitted with daily disposable silicone hydrogel contact lenses. Contact Lens and Anterior Eye 2013;36(3):118-125.

[19] Kutuzov, N., Flyvbjerg, H., Lauritzen, M.. Contributions of the glycocalyx, endothelium, and extravascular compartment to the bloodbrain barrier. Proceedings of the National Academy of Sciences 2018;115(40):E9429-E9438.

[20] Pavlova, V., Paunova-Krasteva, T., Stoitsova, S., Nikolovaa, E.. Distribution patterns of carbohydrates in murine glycocalyx. Biotechnology and Biotechnological Equipment 2015;29(2):357-362.

[21] Hazlett, L.D., Mathieu, P.. Glycoconjugates on corneal epithelial surface: Effect of neuraminidase treatment. Journal of Histochemistry and Cytochemistry 1989;37(8):1215-1224.

[22] Wu, A.M., Wu, J.H., Song, S.C., Tsai, M.S., Herp, A.. Studies on the binding of wheat germ agglutinin (Triticum vulgaris) to O-glycans. FEBS Letters 1998;440(3):315-319.

[23] Bhavanandan, V.P., Katlic, A.W.. The interaction of wheat germ agglutinin with sialoglycoproteins. The role of sialic acid. Journal of Biological Chemistry 1979;254(10):4000-4008.

[24] Monsigny, M., Roche, A.., Sene, C., MagetDana, R., Delmotte, F.. SugarLectin Interactions: How Does WheatGerm Agglutinin Bind Sialoglycoconjugates? European Journal of Biochemistry 1980;104(1):147-153.

[25] Chen, M., Wang, Y., Begley, C.G., Wolosin, J.M.. Synthesis of Rabbit Corneal Epithelial Glycocalyx in vitro. Experimental Eye Research 1994;58(3):267-276.

[26] Mclaughlin, B.J., Barlar, E.K., Donaldson, D.J.. Wheat germ agglutinin and concanavalin a binding during epithelial wound healing in the cornea. Current Eye Research 1986;5(8):601-609.

[27] Ellingham, R.B., Berry, M., Corfield, A.P.. Agarose gel electrophoresis of human tears: Mucin detected. Investigative Ophthalmology and Visual Science 1996;37(3):3902-3902.

[28] Shigeyasu, C., Yamada, M., Akune, Y., Fukui, M.. The effect of rebamipide ophthalmic suspension on ocular surface mucins in soft contact lens wearers. Contact Lens and Anterior Eye 2018;41(4):357-361. 
[29] Rivas, L., Oroza, M.A., PerezEsteban, A., MurubedelCastillo, J.. Topographical distribution of ocular surface cells by the use of impression cytology. Acta Ophthalmologica 1991;69(3):371-376.

[30] Doughty, M.J.. Goblet cells of the normal human bulbar conjunctiva and their assessment by impression cytology sampling. Ocular Surface 2012;10(3):149-169.

[31] Kessing, S.. Mucous gland system of the conjunctiva. A quantitative normal anatomical study. Acta Ophthalmol (Copenh) 1968;Suppl(95):1+.

[32] Garaszczuk, I.K., Iskander, D.R.. Qualitative assessment of tear dynamics with fluorescein profilometry. Contact Lens and Anterior Eye 2017;40(4):208-212.

[33] Occhipinti, J.R., Mosier, M.A., Lamotte, J., Monji, G.T.. Fluorophotometric measurement of human tear turnover rate. Current Eye Research 1988;7(10):995-1000.

[34] Lakkis, C., Brennan, N.A.. Bulbar conjunctival fluorescein staining in hydrogel contact lens wearers. Eye Contact Lens $1996 ; 22(3): 189$.

[35] Guillon, M., Maissa, C.. Bulbar conjunctival staining in contact lens wearers and non lens wearers and its association with symptomatology. Cont Lens Anterior Eye 2005;28(2):67-73.

[36] Korb, D.R., Greiner, J.V., Herman, J.P., Hebert, E., Finnemore, V.M., Exford, J.M., et al. Lid-wiper epitheliopathy and dry-eye symptoms in contact lens wearers. CLAO J 2002;28(4):211-216.

[37] Varikooty, J., Srinivasan, S., Subbaraman, L., Woods, C.A., Fonn, D., Simpson, T.L., et al. Variations in observable lid wiper epitheliopathy (LWE) staining patterns in wearers of silicone hydrogel lenses. Cont Lens Anterior Eye $2015 ; 38(6): 471-476$.

[38] Lakowicz, J.R.. Quenching of Fluorescence. In: Lakowicz, J.R., editor. Principles of Fluorescence Spectroscopy; 3 ed. Boston: Springer; 1999, p. 277-330.

[39] Finnemore, V.M., Korb, D.R., Greiner, J.V., Glonek, T., Herman, J.P.. Fluorescein Dye Concentration as a Factor in Tear Film Fluorescence. In: Sullivan, D., Dartt, D., Meneray, M., editors. Lacrimal Gland, Tear Film, and Dry Eye Syndromes 2. Advances in Experimental Medicine and Biology; chap. vol 438. Boston: Springer; 1998 , p. 875-878.

[40] Maissa, C., Guillon, M., Garofalo, R.J.. Contact Lens-Induced Circumlimbal Staining in Silicone Hydrogel Contact Lenses Worn on a Daily Wear Basis. Eye Contact Lens 2012;38(1):16-26.

[41] Brennan, N.A., Coles, M.L., Ang, J.H.. An evaluation of silicone-hydrogel lenses worn on a daily wear basis. Clin Exp Optom 2006;89(1):18-25.

[42] Brennan, N.A., Coles, M.L.C., Connor, H.R.M., McIlroy, R.G.. A 12-month prospective clinical trial of comfilcon A silicone-hydrogel contact lenses worn on a 30-day continuous wear basis. Cont Lens Anterior Eye 2007;30(2):108-118.

[43] Navascues Cornago, M., Maldonado-Codina, C., Morgan, P.B.. Mechanical Sensitivity of the Human Conjunctiva. Cornea 2014;33(8):855-859.

[44] Ludwig, A.. The use of mucoadhesive polymers in ocular drug delivery. Adv Drug Deliv Rev 2005;57(11):1595-1639.

[45] Khare, A., Singh, I., Grover, K., Pawar, P., Singh, I.. Mucoadhesive Polymers for Enhancing Retention in Ocular Drug Delivery. Progress in Adhesion and Adhesives 2015;2(4):451-484.

[46] Uchino, Y.. The ocular surface glycocalyx and its alteration in dry eye disease: A review. Invest Ophth Vis Sci 2018;59(14):DES157-DES162.

[47] Ryan, D.S., Bower, K.S., Sia, R.K., Shatos, M.A., Howard, R.S., Mines, M.J., et al. Goblet cell response after photorefractive keratectomy and laser in situ keratomileusis. J Cataract Refract Surg 2016;42(8):1181-1189.

[48] Konomi, K., Chen, L.L., Tarko, R.S., Scally, A., Schaumberg, D.A., Azar, D., et al. Preoperative characteristics and a potential mechanism of chronic dry eye after LASIK. Invest Ophthalmol Vis Sci 2008;49(1):168-174

[49] Pult, H., Tosatti, S.G.P., Spencer, N.D., Asfour, J.M., Ebenhoch, M., Murphy, P.J.. Spontaneous Blinking from a Tribological Viewpoint. Ocul Surf 2015;13(3):236-249.

[50] Sterner, O., Karageorgaki, C., Zürcher, M., Zürcher, S., Scales, C.W., Fadli, Z., et al. Reducing Friction in the Eye: A Comparative Study of Lubrication by Surface-Anchored Synthetic and Natural Ocular Mucin Analogues. ACS Appl Mater Interfaces 2017;9(23):20150-20160.

[51] Chen, Y.M., Kurokawa, T., Tominaga, T., Yasuda, K., Osada, Y., Gong, J.P., et al. Study on the sliding friction of endothelial cells cultured on hydrogel and the role of glycocalyx on friction reduction. Adv Eng Mater 2010;12(11):628-636.

[52] Guzman-Aranguez, A., Argueso, P.. Structure and biological roles of mucin-type O-glycans at the ocular surface. Ocul Surf 2010;8(1):8-17.

[53] Ricciuto, J., Heimer, S.R., Gilmore, M.S., Argüeso, P.. Cell Surface O-Glycans Limit Staphylococcus aureus Adherence to Corneal Epithelial Cells. Infect Immun 2008;76(11):5215-5220.

[54] Hamrah, P., Alipour, F., Jiang, S., Sohn, J.H., Foulks, G.N.. Optimizing evaluation of Lissamine Green parameters for ocular surface staining. Eye 2011;25:1429-1434. 